\title{
The BLIXER, a Wideband Balun-LNA-I/Q-Mixer Topology
}

\author{
Stephan C. Blaakmeer, Member, IEEE, Eric A. M. Klumperink, Senior Member, IEEE, \\ Domine M. W. Leenaerts, Fellow, IEEE, and Bram Nauta, Fellow, IEEE
}

\begin{abstract}
This paper proposes to merge an I/Q current-commutating mixer with a noise-canceling balun-LNA. To realize a high bandwidth, the real part of the impedance of all RF nodes is kept low, and the voltage gain is not created at RF but in baseband where capacitive loading is no problem. Thus a high RF bandwidth is achieved without using inductors for bandwidth extension. By using an I/Q mixer with $25 \%$ duty-cycle LO waveform the output IF currents have also $25 \%$ duty-cycle, causing 2 times smaller DC-voltage drop after IF filtering. This allows for a 2 times increase in the impedance level of the IF filter, rendering more voltage gain for the same supply headroom. The implemented balun-LNA-I/Q-mixer topology achieves $>18 \mathrm{~dB}$ conversion gain, a flat noise figure $<5.5 \mathrm{~dB}$ from $500 \mathrm{MHz}$ to $7 \mathrm{GHz}$, IIP2 $=+20 \mathrm{dBm}$ and IIP3 $=-3 \mathrm{dBm}$. The core circuit consumes only $16 \mathrm{~mW}$ from a $1.2 \mathrm{~V}$ supply voltage and occupies less than $0.01 \mathrm{~mm}^{2}$ in $65 \mathrm{~nm}$ CMOS.
\end{abstract}

Index Terms-CMOS integrated circuits, direct conversion, high-linearity mixer, linearity, low noise, low-noise amplifiers, low-power electronics, merged LNA and mixer, microwave integrated circuits, microwave receivers, multi-standard, noise canceling, noise cancellation, radio receivers, $\mathrm{RF}$ transceiver, software defined radio, software radio, UHF integrated circuits, wideband LNA, wideband matching, wideband receiver, wideband RF front-end, zero IF.

\section{INTRODUCTION}

W IDEBAND radio receivers have recently drawn significant research interest, e.g., for emerging software-defined radio (SDR) architectures and ultra-wideband (UWB) communication standards [1]-[3]. Such applications call for radio receivers covering the frequency range from a few hundred $\mathrm{MHz}$ up to about $6 \mathrm{GHz}$ (SDR) or even $10 \mathrm{GHz}$ (UWB). Co-operability with other communication devices (e.g., cellular, WLAN) operating in the same spectrum is mandatory, setting especially stringent demands on the wideband linearity of such a receiver. A single-ended RF input avoids the use of an external broadband balun and its accompanying losses. Compared to a differential input it also requires less switches

Manuscript received April 11, 2008; revised June 21, 2008. Current version published December 10, 2008.

S. C. Blaakmeer is with the University of Twente, CTIT Institute, IC Design group, $7500 \mathrm{AE}$ Enschede, The Netherlands, and also with Axiom IC Twente, Enschede, The Netherlands (e-mail: s.c.blaakmeer@utwente.nl; stephan.blaakmeer@Axiom-IC.com).

E. A. M. Klumperink and B. Nauta are with the University of Twente, CTIT Institute, IC Design group, $7500 \mathrm{AE}$ Enschede, The Netherlands.

D. M. W. Leenaerts is with NXP Semiconductors, Research, Eindhoven, The Netherlands.

Digital Object Identifier 10.1109/JSSC.2008.2004866 to connect the RF input to different RF filters and/or antenna networks [2].

Recently, some wideband balun-LNAs with high linearity have been proposed offering a wideband input match, gain and single-ended to differential conversion [4], [5]. Active mixers have a capacitive input impedance, i.e., the gate of a transistor. When a passive mixer is used, a voltage buffer or transconductance stage is often required between the LNA output and the input of the mixer(s). Also this intermediate stage between LNA and mixer loads the LNA capacitively. Due to this capacitance load, it is challenging to realize high LNA gain over a large bandwidth. Inductive peaking has been used to still achieve $6 \mathrm{GHz}$ bandwidth [3], [6], however we would like to avoid the use of area consuming on-chip inductors in expensive nanometer scale CMOS processes. Moreover, the requirements on linearity in the input stage of a mixer will be higher than for the LNA, because of the voltage gain of the LNA. As the input signal of the mixer is still at a high frequency it is challenging to obtain high linearity there. For instance, negative feedback techniques are not very effective because loop gain is limited at $\mathrm{GHz}$ frequencies.

This paper proposes a solution to the above described problems via a so-called "BLIXER" topology. The topology actually comprises an active balun, LNA and mixer in a single circuit. Without bandwidth extension inductors, it still easily achieves more than $7 \mathrm{GHz}$ bandwidth in $65 \mathrm{~nm}$ CMOS by merging a current commutating I/Q-mixer with a noise-canceling balun-LNA [7]. In this paper we explain the operation of the topology in detail and analyze its gain and noise behavior. Also we compare it to alternative topologies to illustrate its competitive performance.

The paper is structured as follows. Section II quickly reviews recently proposed balun-LNAs to show why it is challenging to simultaneously achieve high gain and high bandwidth. Then we introduce the BLIXER topology in Section III and show how it can simultaneously achieve high gain and high RF bandwidth. In Section IV, we analyze the gain and noise figure of the BLIXER in terms of component design parameters. Section V discusses the circuit implementation and measurement results, while Section VI draws conclusions.

\section{BALUN-LNA GAIN AND BANDWIDTH LIMITATION}

\section{A. Balun-LNA Topology}

Fig. 1 shows a balun-LNA consisting of a parallel operating common gate (CG) and common source (CS) stage. Both stages are cascoded to allow for realizing a high voltage gain via resistor $R_{\mathrm{CG}}$ and $R_{\mathrm{CS}}$. The CG stage realizes wideband input 


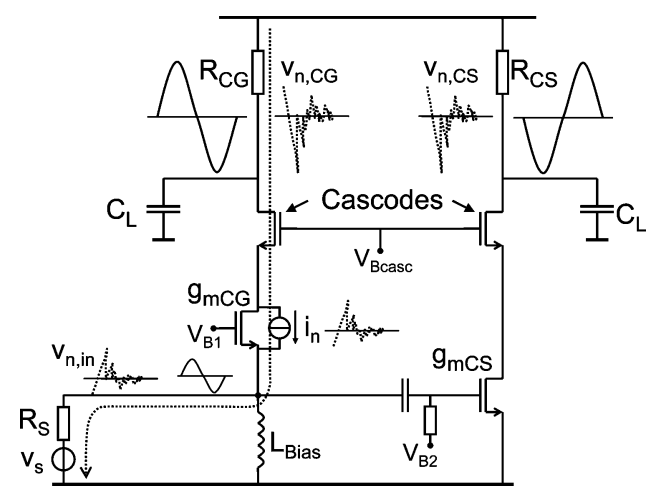

Fig. 1. Balun-LNA of [5] with cascodes added. Signal $v_{s}$ is amplified and converted to a balanced RF output signal, while the noise $i_{n}$ of the impedancematching CG transistor is canceled.

impedance matching and gain, while the CS stage realizes an anti-phase output signal. The circuit can simultaneously achieve noise canceling, distortion canceling and output balancing as discussed in detail in [5]. In a CG stage only, the noise of the CG transistor would be dominant when the input impedance is matched to $R_{S}\left(g_{m C G}=1 / R_{S}\right)$. However, using a properly designed CS stage this noise $\left(i_{n}\right.$ in Fig. 1$)$ can be canceled. The noise current $\left(i_{n}\right)$ generates a noise voltage on the source resistor $\left(v_{n, i n}\right)$ and a larger voltage in anti-phase across $R_{C G}\left(v_{n, C G}\right)$. The input noise voltage $\left(v_{n, i n}\right)$ is amplified by the CS stage to $v_{n, C S}$, which is in-phase and fully correlated with $v_{n, C G}$. For equal CG and CS stage gain, the noise due to the $C G$ transistor is fully canceled at the differential output, while the signal contributions to the output signal add up to create a balanced output. This noise-canceling technique was proposed in [8], [9], while different circuit topologies were generated and compared in [10]. The parallel CS-CG topology (or balun-LNA) was found to be one of the best performing topologies (topology "e") in Figs. 4.22 and 4.23 of [10]). It cancels the noise of the $\mathrm{CG}$ transistor in order to obtain a noise figure (NF) close to or lower than $3 \mathrm{~dB}$ [3]-[6], [9], [11]-[13]. To achieve this low NF, the impedance of the CS stage needs to be scaled down $n$ times with respect to the CG stage, where $n$ is typically in the order of 4 (see Fig. 2).

The CG stage is biased using an external inductor $\left(L_{\text {bias }}\right)$ to obtain low-noise operation and save valuable voltage headroom. Depending on the application this inductor can either be very large (RF-choke) for wideband operation, or smaller and well-defined to tune to a dedicated band and realize RF pre-filtering. Especially for software defined radio transceivers this is a realistic approach, as some RF pre-filtering is commonly needed to make the linearity requirements of a CMOS transceiver feasible [14]. Using an external inductor at the input of a CMOS chip allows for realizing external re-configurability and generally the quality factor of the off-chip inductors is higher than on-chip realizations.

\section{B. Achievable Gain and Bandwidth}

The voltage gain of transistors in modern CMOS processes is low. Cascoding is an effective method to increase the voltage gain. The output resistance of the transconductor (input transistor plus cascode) is increased, so that increasing $R_{C G}$ and

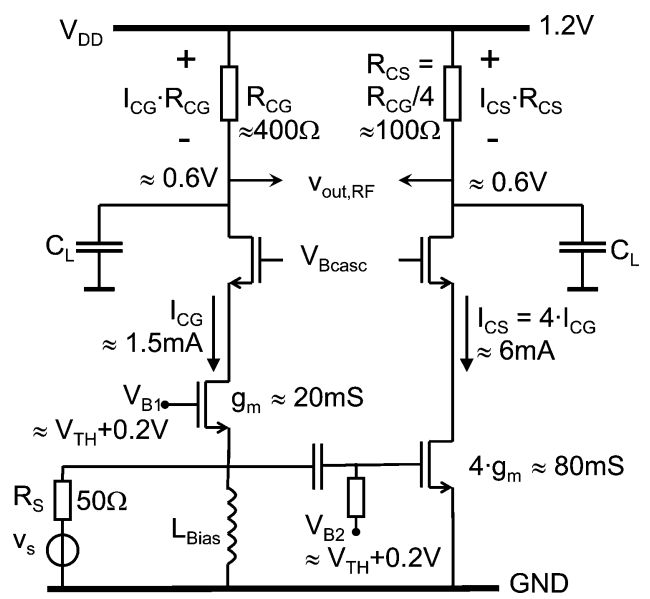

Fig. 2. Practical balun-LNA design for $n=4$ illustrating typical component dimensioning and biasing in a $65 \mathrm{~nm}$ CMOS technology.

$R_{C S}$ in Fig. 1 still improves the voltage gain. Next to this, the cascodes lower the effective input capacitance, as the Miller effect is reduced (most important for the CS transistor, as it is $n$-times wider than the CG transistor). This helps to improve the bandwidth over which good input impedance matching is achieved.

Let us now consider the gain and bandwidth of the balun-LNA, neglecting the effect of body effect for simplicity. In Fig. 2 a typical design in a $65 \mathrm{~nm}$ CMOS technology is shown. The transconductance of the CG transistor is $20 \mathrm{mS}$ to realize impedance matching to a $50 \Omega$ source. This requires about $1.5 \mathrm{~mA}$ of biasing current for a transistor with $W / L=90 \mu \mathrm{m} / 0.06 \mu \mathrm{m}$ at a moderate overdrive voltage of $V_{G T} \approx 0.2 \mathrm{~V}$. We design the voltage drop across the load resistor to be $0.6 \mathrm{~V}$, half of the $1.2 \mathrm{~V}$ supply voltage. This leaves $0.6 \mathrm{~V}$ for the sum of $V_{D S}$ 's of the input and cascode transistor, which is sufficient to keep each MOS transistor in saturation $\left(V_{D S}>V_{G T}\right)$. With $1.5 \mathrm{~mA}$ bias current in the CG stage and $0.6 \mathrm{~V}$ headroom, the CG load resistor $\left(R_{C G}\right)$ becomes $400 \Omega$. If we use the same components and biasing in the CS stage, and neglect the body effect, then DC-bias and modulus of the gain of the stages are equal. Since the CG noise is cancelled, the CS noise dominates in the LNA. To achieve acceptable noise, the impedance level in the CS stage is assumed to be scaled down by 4 times $(80 \mathrm{mS}$ and $100 \Omega$ ). This does not affect the DC-voltages and voltage gain. With $g_{m, C G}=20 \mathrm{mS}$ and $R_{C G}=400 \Omega$, the voltage gain of the CG stage is

$$
A_{v, C G}=g_{m C G} \cdot R_{C G}=20 \cdot 10^{-3} \cdot 400=8 .
$$

As the gain of the CS stage is equal but with opposite sign, the total voltage gain of the CG-CS LNA from single-ended input to differential output is 2 times higher:

$$
A_{v, C G-C S}=2 \cdot A_{v, C G}=16 \Leftrightarrow 24 \mathrm{~dB} .
$$

A high voltage gain can thus be obtained by using this parallel connection of a CG and a CS stage. However, the bandwidth associated with this high gain is limited due to the dominant pole at the output of the CG stage. For a $3 \mathrm{~dB}$ bandwidth of 
$10 \mathrm{GHz}$, the loading capacitance $\left(C_{L}\right)$ is most critical at the $\mathrm{CG}$ side and should be smaller than

$$
C_{L}<\frac{1}{2 \pi \cdot R_{C G} \cdot f_{-3 \mathrm{~dB}}}=\frac{1}{2 \pi \cdot 400 \cdot 10 \cdot 10^{9}}=40 \mathrm{fF} .
$$

The load capacitance is the sum of the input capacitance of the next stage (input stage of the mixer) and the capacitance of the cascode transistor. For a cascode transistor with $W / L=$ 90/0.06 (same size as the CG transistor), the capacitance of the only the cascode is already close to the maximal allowed capacitance:

$$
C_{\text {Casc }} \approx C_{G D O}+C_{J D B}=15 f+22 f=37 \mathrm{fF}
$$

where the two dominant capacitances seen at the drain of the cascode are the gate-drain overlap capacitance $\left(C_{G D O}\right)$ and the drain-bulk junction capacitance $\left(C_{J D B}\right)$. This means that no (capacitive) load can be driven when a $3 \mathrm{~dB}$ bandwidth in the order of $10 \mathrm{GHz}$ is required. This clearly shows that there is a bandwidth problem at the load of the CG side for high enough voltage gain. As one of the goals in this design was to avoid the use of on-chip inductors, no inductive peaking techniques [3], [6] will be used to broaden the bandwidth. The BLIXER topology we propose in the next section achieves high bandwidth without requiring on-chip inductors.

\section{THE BLIXER TOPOLOGY}

\section{A. The Basic BLIXER Topology}

In Fig. 3, the principle of the BLIXER topology is shown. Compared to the balun-LNA of Fig. 1, the same CG and CS transistors are used while the cascode transistors are now part of a current commutating mixer. Instead of one, both the CG and the CS side have now two cascode (or mixer) transistors, which are periodically switched on and off, with frequency $f_{\mathrm{LO}}$. The current from the CG and CS transistor can always flow towards the loads (discussed in Section III-B), as at any moment in time one of its mixer (cascode) transistors is active, i.e., the LO signal has 50\% duty-cycle. At the drains of the mixer the signal of interest is down converted to an intermediate frequency (IF) which is much lower than the RF frequency. In the BLIXER topology, the capacitance at the loads sets the IF bandwidth, instead of the $R F$ bandwidth in case of the balun-LNA. As the IF bandwidth is much lower than the RF bandwidth, the capacitance at the loads can be much higher. Furthermore, the capacitive load of the next stage can be absorbed into the capacitance of the IF filter. The bandwidth problem at the load of the CG stage, described in the previous section, is thus solved without requiring inductors for bandwidth extension.

There are only three RF signal nodes in the BLIXER topology: the input node and the two drain nodes of the CG and CS transistor. This means that in the complete down-converter there are only three nodes that can limit the RF bandwidth. For impedance matching, the real part of the input impedance of the circuit equals $R_{S}(50 \Omega)$. When the real part of the impedances at the two drains nodes is low (indicated in Fig. 3), the bandwidth at these nodes can be high. For switch transistors equal to the cascode transistors in Fig. 2, the real parts at the drains of the CG and CS transistors are $50 \Omega$ and $12.5 \Omega$, respectively, $\left(=1 / g_{m}\right.$

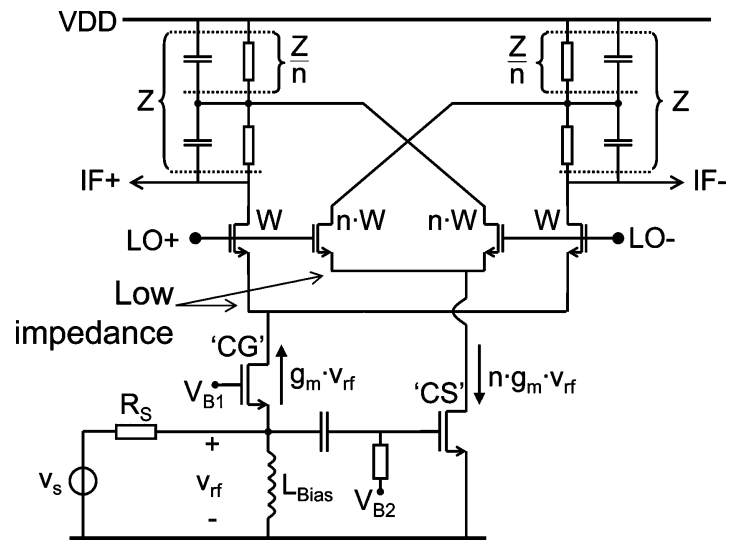

Fig. 3. Basic BLIXER topology consisting of the balun-LNA core of Fig. 1 with doubled cascode transistors driven by an LO implementing down-conversion mixing to IF.

of mixer transistors). Thus, all RF node impedances are in the order of $50 \Omega$ or lower, allowing for high bandwidth.

The CG and CS transistors can be considered as a transconductor converting $v_{\mathrm{rf}}$ to currents $g_{m} \cdot v_{\mathrm{rf}}$ and $n \cdot g_{m} \cdot v_{\mathrm{rf}}$ (see Fig. 3). These currents are largely conveyed to the IF output via the switched cascode transistors, provided the real input impedance of these transistor is lower than the parasitic capacitance to ground. Only modeling gate-source capacitances, the RF bandwidth limitation due to the switched cascode transistors

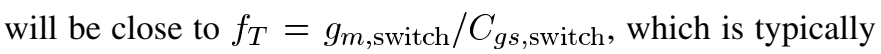
an order of magnitude higher than for the balun-LNA with a voltage gain in the order of $20 \mathrm{~dB}$.

\section{B. Noise Canceling at IF Instead of at RF}

Consider now the real part of the load impedance, which consists of four resistors instead of two in the balun-LNA. A bit more complex load is used in order to maintain the noise-canceling conditions in both LO switch positions. In Fig. 4, the active part of the circuit is shown when LO+ is high. As in Fig. 1, the CG side has $Z$ as load, while the load in the $n$ times scaled CS side is $Z / n$. The IF output voltage is sensed at the combined CG and CS load, where the lower capacitance of the current-less branch effectively works as level shifter. As the impedance of the CS side is $n$ times lower than for the CG side, it has a low noise contribution and still the gain of the CG and the CS sides is equal. As the gain of both sides is equal, the noise of the CG transistor is canceled in the same way as in the balun-LNA of Fig. 1 Note that in the BLIXER topology the noise canceling takes place after the frequency (down) conversion i.e., at IF. The noise-canceling down-converter in [15] also has this property. However, it is much less power efficient as cascaded stages instead of cascoding (i.e., stacking of transistors with current re-use) was used, while also high overdrive voltages were used to push the bandwidth.

\section{The I/Q-BLIXER Topology}

Quadrature outputs are required in order to implement a lowor zero-IF receiver. It is possible to create quadrature outputs starting with signal currents $i$ and $-i$ from a differential pair, by connecting two switching pairs to each current, and using a sine 


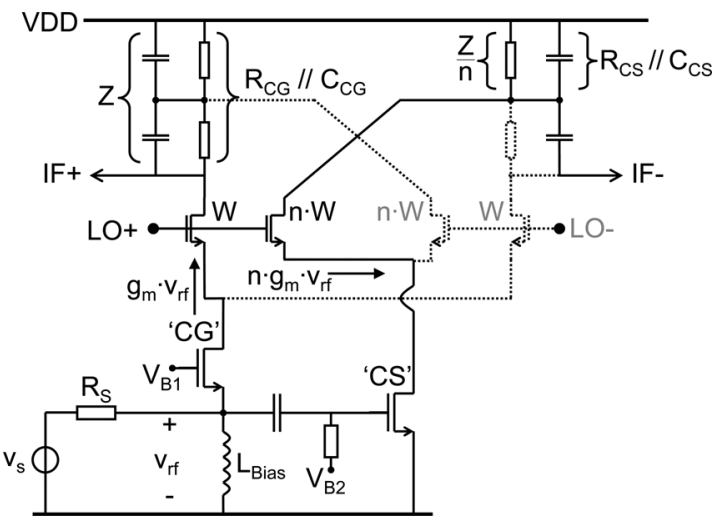

Fig. 4. Equivalent circuit of the BLIXER when $\mathrm{LO}+$ side is active. As in Fig. 1, the current of the CG stage flows into its load impedance $R_{C G}\left(/ / C_{C G}\right)$, and the CS current flows into $R_{C S}\left(/ / C_{C S}\right)$, which impedance is $n$ times smaller.

and cosine wave to drive the two pairs [16], [17]. In principle this is also possible with the asymmetrical balun-LNA currents $i$ and $-4 i$, provided that the switch transistors and load network are scaled as shown in Fig. 5. We will refer to this circuit as the I/Q-BLIXER. Simulations show that it is possible to use sine and cosine LO signals in I/Q-BLIXER, however this seems mainly attractive for narrowband receivers like in [16] and [17]. For narrowband receivers, the required sine and cosines can be generated via a quadrature $\mathrm{VCO}$ with relatively small tuning range. For multi-standard receivers or software defined radio we want much wider tuning range. Digital frequency synthesis methods exploiting Moore's law are then preferred [18]. However, using two square waves with $50 \%$ duty cycle with a relative delay of $1 / 4$ period gives $25 \%$ overlap between LO I and LO Q pulses. Using a $25 \%$ duty-cycle, this overlap can be avoided and it is possible to "isolate" the I- and Q- current-path in time. Hence, the full $g_{m}$ of the input devices is available to either the I- or Q-output of the circuit which is beneficial for the conversion gain. Furthermore, we will show in the next section that, without compromising the voltage headroom, it is possible to double the value of the IF load resistors to increase the gain.

\section{Conversion Gain and Voltage Drop Load Resistors}

The voltage conversion gain from single-ended input to differential output of the BLIXER topology (Fig. 3) can be calculated as

$$
\begin{aligned}
G_{\text {BLIXER }} & =\frac{2}{\pi} \cdot\left(g_{m C G} \cdot R_{C G}+g_{m C S} \cdot R_{C S}\right) \cdot 2 \cdot \frac{1}{2} \\
& =\frac{2}{\pi} \cdot\left(g_{m C G} \cdot R_{C G}+g_{m C S} \cdot R_{C S}\right)
\end{aligned}
$$

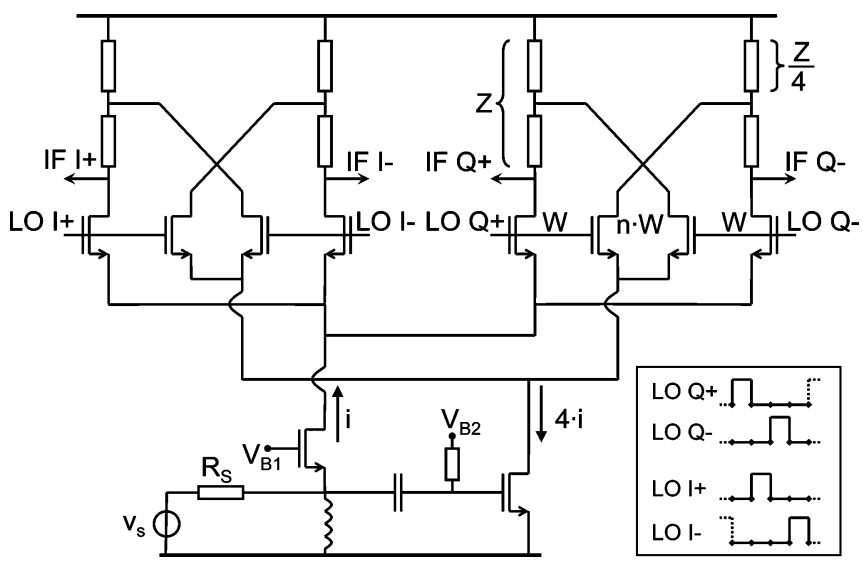

Fig. 5. I/Q-BLIXER driven by LO waveforms with $25 \%$ duty cycle, which allows for sharing currents $i$ and $4 \cdot i$ by an I- and Q- mixer.

where, after the first equal sign, the factor $2 / \pi$ equals the fundamental Fourier component of a $50 \%$ duty-cycle (LO)-signal toggling between 0 and 1 . The term between brackets is the combined voltage gain of the CG and CS side. The factor 2 is because a differential LO signal is used, and the final factor is because only the down converted half of the signal is used, as shown in (6) at the bottom of the page.

Similarly, the voltage gain of the I/Q-BLIXER (Fig. 5) with $25 \%$ duty-cycle LO equals

$$
G_{\text {IQBLIXER }}=\frac{\sqrt{2}}{\pi} \cdot\left(g_{m C G} \cdot R_{C G}+g_{m C S} \cdot R_{C S}\right)
$$

where the factor $\sqrt{2} / \pi$ equals the fundamental Fourier component of a $25 \%$ duty-cycle LO signal.

Compared to the BLIXER, the conversion gain of the I/QBLIXER is lower due to the lower duty-cycle, assuming the same $g_{m}$ 's and load resistors are used. However, in the I/Q-BLIXER larger load resistors can be used, as shown below.

The average (or DC) voltage drop across the load resistors in the BLIXER is

$$
\overline{V_{\text {Load }}}=\frac{1}{2}\left(I_{C G} \cdot R_{C G}+I_{C S} \cdot R_{C S}\right) .
$$

This is because with $50 \%$ duty-cycle LO, half of the period the CG transistor bias current $\left(I_{C G}\right)$ flows through $R_{C G}$ and in the other half the CS bias current $\left(I_{C S}\right)$ a current flows through $R_{C S}$.

In the I/Q-BLIXER, the DC-voltage drop across the load equals

$$
\overline{V_{\text {Load }}}=\frac{1}{4}\left(I_{C G} \cdot R_{C G}+I_{C S} \cdot R_{C S}\right)
$$

$$
\begin{aligned}
s(t)= & \cos \left(\omega_{\text {Signal }} t\right) \cdot \cos \left(\omega_{\mathrm{LO}} t\right) \\
= & \frac{1}{2} \cdot \cos \left(\left(\omega_{\text {Signal }}-\omega_{\mathrm{LO}}\right) t\right) \rightarrow \text { down converted, used IF }- \text { signal } \\
& +\frac{1}{2} \cdot \cos \left(\left(\omega_{\text {Signal }}+\omega_{\mathrm{LO}}\right) t\right) \quad \rightarrow \text { up converted, removed by IF }- \text { filter. }
\end{aligned}
$$




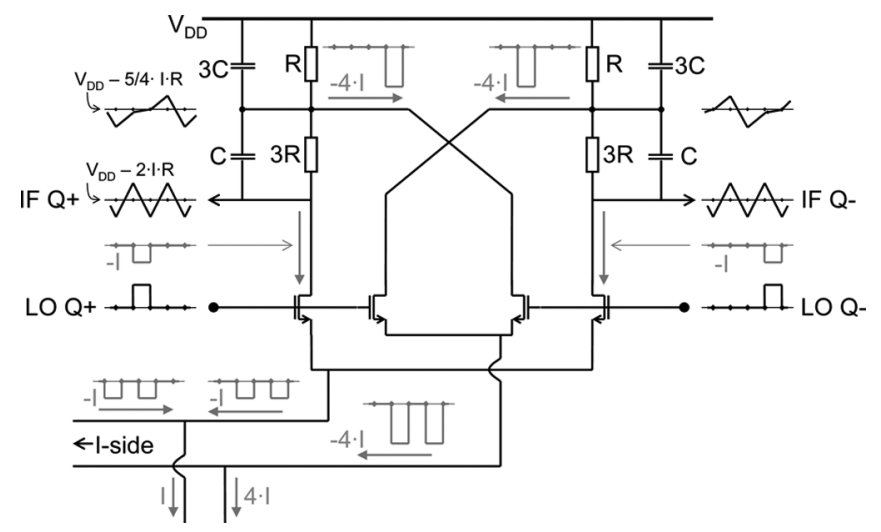

Fig. 6. Q-side of the I/Q-BLIXER showing the branch currents and node voltages.

as with $25 \%$ duty-cycle the CG and CS bias current flow only for $1 / 4$ of the period through one of the loads (the waveforms are shown in Fig. 6). The capacitors in the load average these pulses of current and filter out the high-frequency components.

Comparing (8) with (9) we see that, assuming equal transconductor bias currents $\left(I_{C G}\right.$ and $\left.I_{C S}\right)$ and equal voltage drop across the load, we can double the load resistors $\left(R_{C G}\right.$ and $\left.R_{C S}\right)$ in the I/Q-BLIXER. This doubling of the load resistors $(+6 \mathrm{~dB})$ compensates the gain reduction caused by the lower duty-cycle $(-3 \mathrm{~dB})$ and results in $3 \mathrm{~dB}$ more conversion gain for the I/Q-BLIXER compared to a $50 \%$ duty cycle BLIXER.

A numerical example clarifies this further. Using exactly the same components and biasing as the balun-LNA of Fig. 2 for the BLIXER results in the same average voltage drop across the load resistors $(0.6 \mathrm{~V})$. Using (5) the conversion gain equals: $G_{\text {BLIXER }}=20 \mathrm{~dB}$, which is about $4 \mathrm{~dB}$ lower $(2 / \pi)$ than the gain of the balun-LNA. In the I/Q-BLIXER we can double the load resistors, i.e., $R_{C G}=800 \Omega$ and $R_{C S}=200 \Omega$, for equal load voltage drop. This results in an I/Q-BLIXER with a gain of $G_{\text {IQBLIXER }}=23 \mathrm{~dB}$, which is only $1 \mathrm{~dB}$ lower than the balun-LNA, and $3 \mathrm{~dB}$ higher than the BLIXER.

\section{E. Similar Topologies in Literature}

In [19], a MiCROMIXER cell with single-ended input and differential output was proposed. It uses a bipolar equivalent of the CG-CS input stage. However the possibility of noise canceling was not recognized nor exploited. Two of these mixer-cells and an additional LNA would be needed for I/Q operation with acceptable NF.

A merged LNA and I/Q-mixer was published in [16]. However, this design is narrowband, uses 3 on-chip inductors and requires a differential RF input signal. In contrast, the I/Q-BLIXER topology does not require on-chip inductors, achieves wideband single-to-differential operation and I/Q-mixing in one circuit cell.

In [20], a "merged CMOS LNA and Mixer exploiting noise cancellation" is proposed, which seems related at first sight ("merged" and "noise cancellation"). However, a closer look shows this combination actually uses a folded mixer with separate bias currents for the LNA and mixer core (no current re-use). Also the used noise canceling is of another type rendering partial canceling. This circuit has no I/Q outputs, requires a differential input (and external balun) and uses four on-chip inductors. Still, the circuit also uses down-conversion via current commutation directly on the output current of a CG input stage, and is in that sense related. We will compare its performance at the end of the paper (see Table I).

\section{F. Attractive Properties of BLIXER Topologies}

Apart from the bandwidth advantages, the BLIXER topology has some other advantageous properties with respect to linearity, balancing of the output and power consumption, as discussed below.

Similar as the noise, the distortion generated by the CG transistor is canceled [4], [5], [11] and the load resistors are linear. If the switch transistors are well switched, the mixer transistors convey the output current of the CG-CS stage to the output without much distortion (assuming $1 / \mathrm{g}_{\mathrm{m}}$ is significantly lower than the somewhat nonlinear output impedance of the CG/CS transistors). This means that the only remaining source of nonlinearity in the BLIXER is the V-to-I conversion of the CS transistor. In a traditional cascade of voltage-gain LNA and active mixer, the mixer input transistors experience almost an order of magnitude more voltage swing that the RF input, and hence often limit the overall linearity. As the CS transistor of the BLIXER directly senses the RF input voltage without pre-amplification, relatively high linearity can easily be obtained.

Compared to the balun-LNA of Fig. 1, the BLIXER also improves on the quality of the balance of the output signal. This is because the output load network is completely symmetrical in the BLIXER, instead of the $Z$ and $Z / n$ in the balun-LNA. Moreover, both the CG and the CS stage contribute to both output signals (each half of the time). Even if the ratio of $g_{m, C G}$ and $g_{m, C S}$ is not exactly equal to the ratio of the $C S$ - and $C G$-load impedances $(1: n)$, still the gain balancing at the IF output is unaffected. Such a mismatch does make the noise canceling less perfect, but as shown in [8], [10] noise canceling is robust for such errors as $\pm 20 \%$ mismatch still only gives 0.1 times the noise of the CG transistor (without canceling).

The power efficiency of the BLIXER is also attractive as the bias-current of the balun-LNA is re-used to realize the mixer functionality. Especially when the I/Q configuration is used, a complete $\mathrm{I} / \mathrm{Q}$ receiver with high conversion gain is obtained re-using the power of balun-LNA. Of course, the LO drivers do require additional power, but this power will scale down in future technologies with Moore's law.

\section{IMPLEMENTATION AND SIMULATIONS}

In the following sections we will discuss the actual chip implementation of the BLIXER in CMOS $65 \mathrm{~nm}$ technology and we will evaluate its performance via simulations, comparing to the balun-LNA and basic BLIXER.

\section{A. Input Transconductor Implementation}

The implementation of the input transconductor is shown in Fig. 7. As discussed in Section III-F the CS stage determines the linearity of the circuit. In order to obtain high (third-order) linearity the CS transistor should be biased with a high $V_{G T}$. However, at a high $V_{G T}$, the $g_{m} / I_{D}$ of a transistor is low, which 


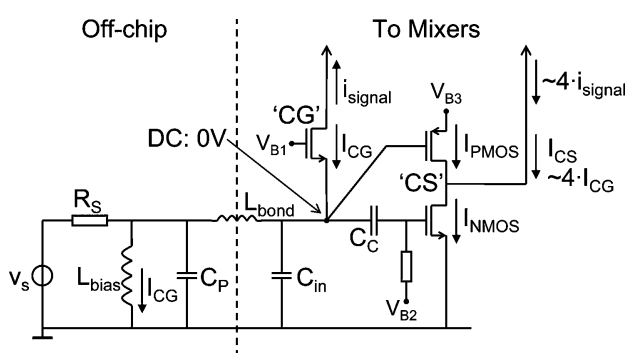

Fig. 7. Implementation of the input stage. The CS transistor is split is a PMOS and an NMOS transistor.

means that a large bias current is required to reach a certain $g_{m}$. This reduces the voltage headroom as this bias current has to flow through the loads. Therefore, the CS stage is implemented using a PMOS and a NMOS transistor. Both the NMOS and PMOS can be biased at a high $V_{G T}$ for high third-order linearity, whereas also the second-order distortion can be low in this inverter-type of circuit. The effective $g_{m}$ of this inverter based CS stage is designed to be about 4 times higher than the effective $g_{m}$ of the $\mathrm{CG}$ transistor. Also the DC-output current the CS stage inverter $\left(I_{C S}=I_{\mathrm{NMOS}}-I_{\mathrm{PMOS}}\right)$ is designed to be approximately 4 times higher than the bias current of the CG transistor. Besides the high linearity, another advantage of the inverter-type CS is that the PMOS transistor can be DC-coupled to the input, which reduces the AC-coupling related signal-loss to the input of the CS stage.

The CG transistor is biased using an inductor which puts the source of the CG transistor at DC-ground, as indicated in Fig. 7. Because the biasing of the CG transistor does not require any DC-voltage drop, the voltage headroom of the CG and CS side is equal. A large bias inductor $\left(L_{\text {bias }} \sim 40 \mathrm{nH}\right)$ can be used as it is placed off-chip. This large inductor allows for good matching in the lower frequency range. In the higher frequency range, the parasitic capacitance of the inductor $\left(C_{\mathrm{par}}\right)$ dominates. Together with the total bondwire inductance and input capacitance ( $L_{\text {bond }}$ and $C_{\text {in }}$ ) a broadband-matching $\pi$-network is formed, which results in impedance matching up to high frequencies.

\section{B. Switches and Load Implementation}

The width of the switches switching the CG current was chosen at $40 \%$ of the width of the $\mathrm{CG}$ transistor. The reason for choosing a small(er) width is to reduce the input capacitance of the switches. The area of a transistor determines its $1 /$ f-noise. In order to lower the $1 /$ f-noise contribution of the switch transistors, their area was increased by setting the length to $100 \mathrm{~nm}$, instead of using minimum length $(65 \mathrm{~nm})$ devices. As overlap and junction capacitances dominate the capacitance of transistors with small length, increasing the length increases the input capacitance of the switches only slightly. The CS switches are 4 times wider than the CG switches.

The impedance ratio of the CG and CS part of the load was designed equal to the ratio of effective $\mathrm{CS}$ and $\mathrm{CG}$ transconductance, which is nominally slightly lower than 4 . The bandwidth of both parts of the loads was designed in the order of $300 \mathrm{MHz}$, enough to handle UWB signals in a zero-IF architecture.

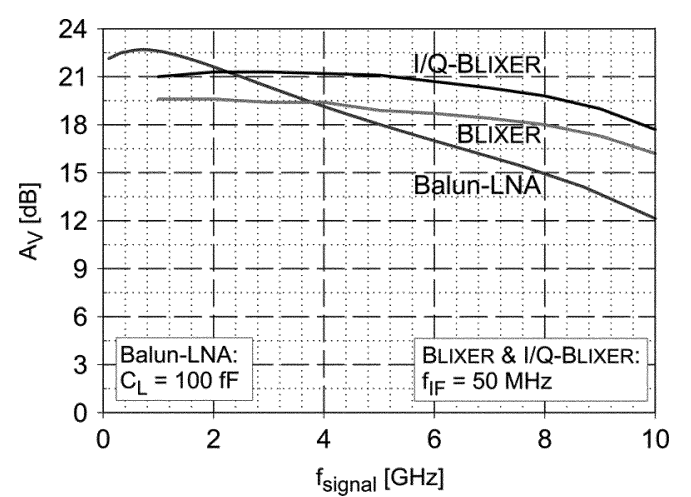

Fig. 8. Gain of the balun-LNA, basic BLIXER (50\% LO duty-cycle) and I/QBLIXER (25\% LO duty-cycle). Note that the BLIXERs have much higher bandwidth $(9.5 \mathrm{GHz})$.

\section{Conversion Gain and Noise Figure Simulations}

In this section we will evaluate the gain and noise figure of the BLIXER and the I/Q-BLIXER and compare it to the standalone balun-LNA. The aim of this comparison is to show that the BLIXERs can have more bandwidth, at practical values for the gain and noise figure. In order to make a fair comparison, all three circuits use the input stage described in Section IV-A, and the dimensions of the (switched or biased) cascode transistors are equal. Choosing this, the bias current of the three circuits cores is equal. However, the BLIXER and I/Q-BLIXER have more functionality than the balun-LNA, as these circuits also perform (I/Q) down-conversion.

As discussed in Section III-D, the load impedance of the BLIXER and the balun-LNA needs to be half the load impedance of the I/Q-BLIXER, in order to keep the average voltage drop across the loads equal in all three cases. The loads in the balun-LNA and BLIXER are halved by placing two I/Q-BLIXER loads in parallel. The switches of the BLIXER and I/Q- BLIXER are driven with ideal $50 \%$ and $25 \%$ duty-cycle LO signals respectively (rise and fall time $1 \%$ of the LO period).

Fig. 8 shows the voltage conversion gain of the BLIXERs as a function of the input signal frequency for an IF of $50 \mathrm{MHz}$. Also the voltage gain versus signal frequency of the balun-LNA, loaded with $100 \mathrm{fF}$ on both outputs, is shown. Clearly, the bandwidth of the BLIXERs is much higher $(\sim 9.5 \mathrm{GHz})$ than the bandwidth of the balun-LNA $(\sim 3.5 \mathrm{GHz})$. As expected, the (low frequency) conversion gain of the I/Q-BLIXER is about $1 \mathrm{~dB}$ lower than the gain of the balun-LNA.

Fig. 9 shows the Noise Figures of the three topologies. The single side band (SSB) noise figure of the BLIXER is $\sim 4 \mathrm{~dB}$ higher than the NF of the balun-LNA. This can be expected based on the conversion-loss of the mixing action $(2 \pi$ or $-4 \mathrm{~dB})$. In contrast to the BLIXER, the I/Q-BLIXER has I- and Q-outputs which can be combined to reject the noise of the image band. Therefore, the relevant measure of NF for the I/Q-BLIXER is the double side band (DSB) NF. Fig. 9 shows that compared to the standalone balun-LNA, the NF of the I/Q-BLIXER increases with only 1-2 dB.

\section{Effects of Non-Ideal LO Signals}

For higher LO frequencies, it becomes increasingly challenging to generate a $25 \%$ duty-cycle LO signal with sufficiently 


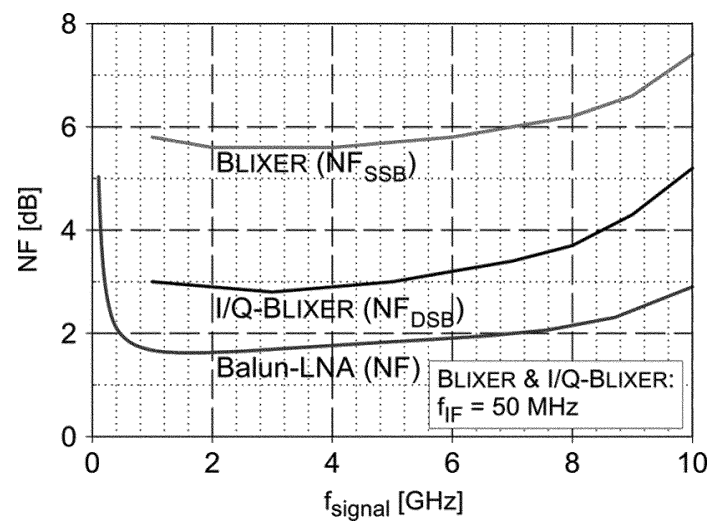

Fig. 9. Comparison of the noise figure of the balun-LNA, basic BLIXER and I/Q- BLIXER. $\mathrm{NF}_{\mathrm{DSB}}$ is used for the I/Q-BLIXER as it has quadrature outputs.

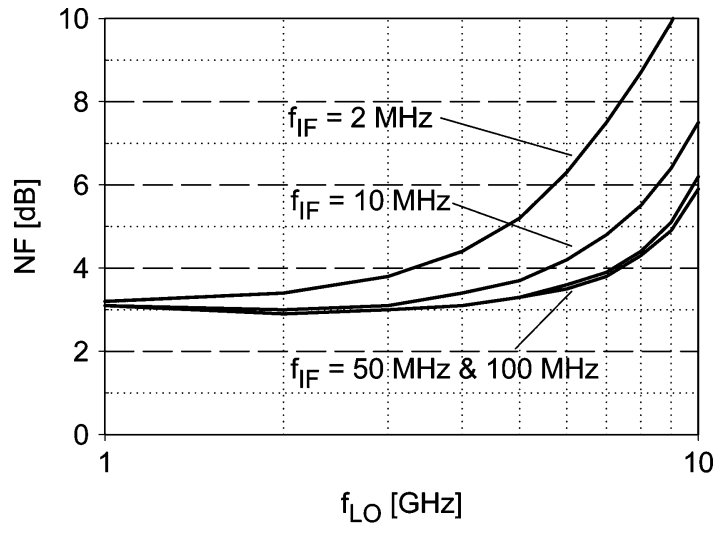

Fig. 10. Effect of LO pulse with finite rise and fall time in the I/Q-BLIXER on the NF versus the LO frequency for different IF frequencies.

low rise and fall times. Simulations on the I/Q-BLIXER are performed to investigate the effects of finite rise and fall times. An ideal voltage source generating a $25 \%$ duty-cycle LO signal with $10 \%$ rise and fall time is supplied to a cascade of two inverters, for each of the four LO phases. The inverters used for this simulation are equal to the inverters used in the final stage of the LO driver in the complete I/Q-BLIXER realization. The size of second inverter is twice the size of the first inverter, and is designed to drive the $\mathrm{LO}$ switches. The inverters limit the rise and fall time to about 25-30 ps, which causes narrower pulses and lowers pulse-amplitude for higher $\mathrm{LO}$ frequencies.

Fig. 10 shows that for higher $\mathrm{LO}$ frequencies, mainly the NF at low IF frequencies ( $2 \mathrm{MHz}$ ) is affected. The increase in NF at low IF frequencies is due to an increased sensitivity to $1 / \mathrm{f}$-noise of the $\mathrm{LO}$ switches and the $\mathrm{LO}$ driver (inverter) transistors. The reduction of conversion gain compared to the case with ideal LO drive is smaller than $1 \mathrm{~dB}$ (not shown as this is only a minor effect).

\section{IC IMPLEMENTATION AND MEASUREMENTS}

Fig. 11 shows an overview of the I/Q-BLIXER test chip. The RF input is single-ended while the IF I- and Q-outputs are differential. The single-ended external oscillator signal is converted into a differential signal on-chip and subsequently divided by 2 to generate in-phase (I) and quadrature (Q) LO signals. The output of the divide-by-2 is amplified to rail-to-rail

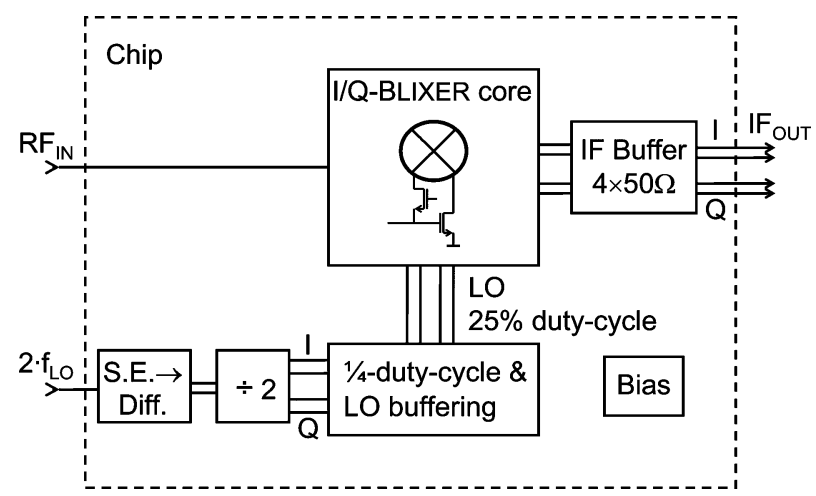

Fig. 11. Overview of the test chip. The LO is derived from an external clock via a single-to-different converter and divide-by- 2 to generate quadrature phases.

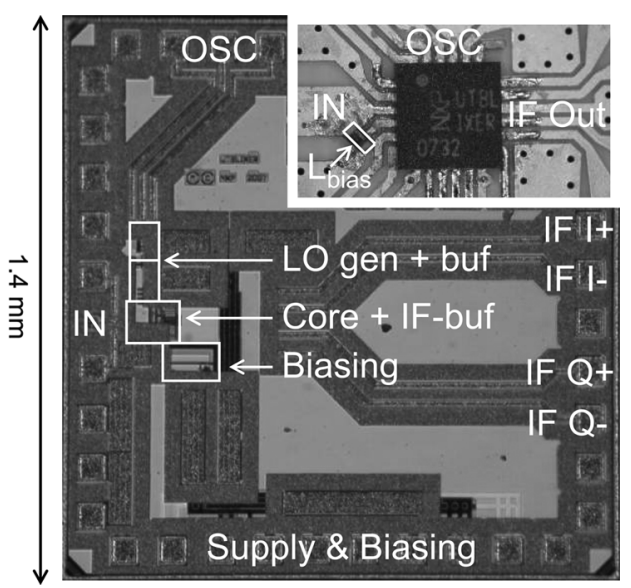

Fig. 12. Chip micrograph and PCB detail showing packaged sample.

swing to obtain a 50\% duty-cycle LO signal. The required $25 \%$ duty-cycle is generated using an AND operation on the I- and Q-signals. The I/Q-BLIXER core is implemented as described in Sections IV-A and Sections IV-B. The differential IF outputs of the I/Q-BLIXER are buffered to $50 \Omega$ for measurement purposes using four source-followers.

The circuit was fabricated in a baseline $65 \mathrm{~nm}$ LP CMOS technology and a standard $1.2 \mathrm{~V}$ supply was used. The die photo and a detail of the PCB are shown in Fig. 12. The I/Q-BLIXER core measures less than $0.01 \mathrm{~mm}^{2}$. The measurements were performed on packaged, PCB-mounted samples.

The measured voltage conversion gain is $19 \mathrm{~dB}$ with and the IF bandwidth is $400 \mathrm{MHz}$, as shown in Fig. 13. The DSB NF of the I/Q-BLIXER at a $3 \mathrm{GHz}$ LO frequency is around $4.5 \mathrm{~dB}$ and flat over the IF bandwidth. The wideband RF performance is shown in Fig. 14. The gain remains flat within $1 \mathrm{~dB}$ up to $7 \mathrm{GHz}$. From 1 to $6 \mathrm{GHz}$, the NF is below $5 \mathrm{~dB}$, using a fixed IF of $50 \mathrm{MHz}$. Note that this NF includes the PCB losses. Above $7 \mathrm{GHz}$, the circuit generating the $25 \%$ duty-cycle LO signals fails and the gain and NF could not be determined. The $S_{11}$ is below $-10 \mathrm{~dB}$ up to $7 \mathrm{GHz}$.

The wideband linearity was measured using a two tone test. To assess IIP2 and IIP3 without filtering effects, the input tones and the intermodulation products should fall within the flat part of the conversion gain versus frequency curve (Fig. 14). In a wideband system a high spacing between the two test tones can 


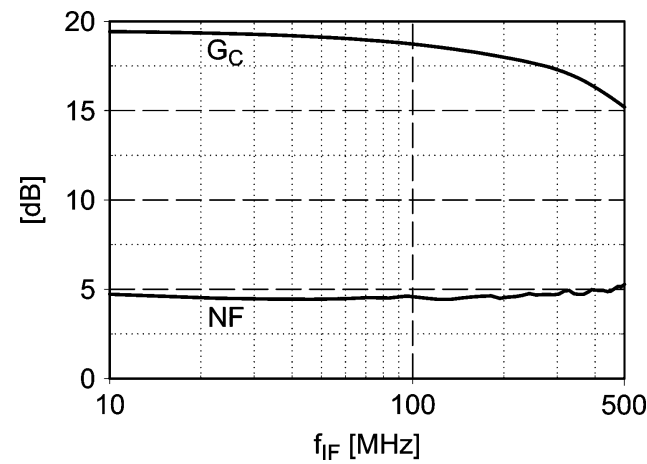

Fig. 13. Conversion gain $\left(G_{C}\right)$ and $\mathrm{NF}$ for $f_{\mathrm{LO}}=3 \mathrm{GHz}$.

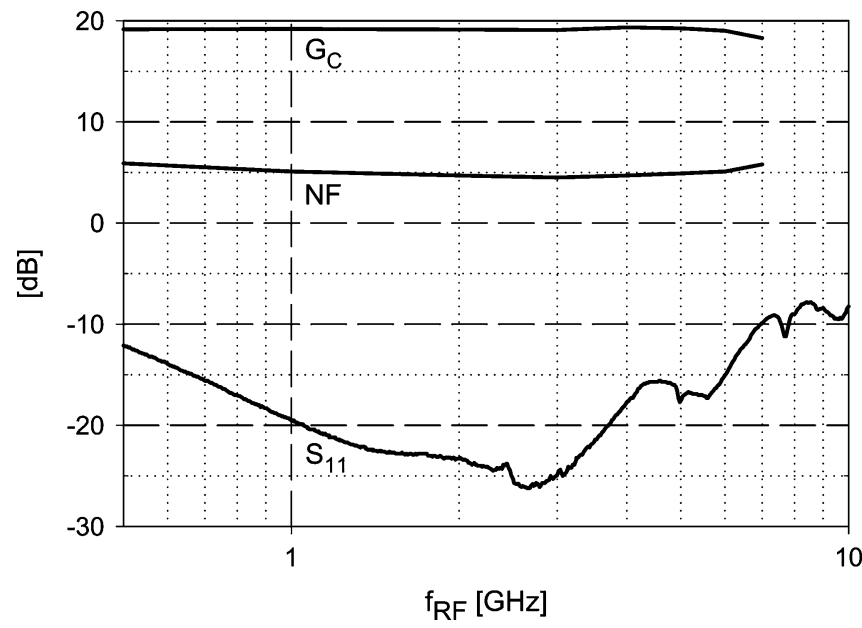

Fig. 14. Conversion Gain $\left(G_{C}\right), \mathrm{NF}$ and $\mathrm{S}_{11}$ versus $\mathrm{RF}$ input-frequency $\left(f_{I F}=50 \mathrm{MHz}\right)$.

be used. Two tones at $5.2 \mathrm{GHz}$ and $5.7 \mathrm{GHz}$, which represents two IEEE 802.11a interferers, gives a 3rd order intermodulation product at $4.7 \mathrm{GHz}$. Using an $\mathrm{LO}$ frequency of $4.6 \mathrm{GHz}$ the measured IIP $3=-3 \mathrm{dBm}$. The IIP2 equals $+20 \mathrm{dBm}$, using $2.4 \mathrm{GHz}(802.11 \mathrm{~b} / \mathrm{g})$ and $5.7 \mathrm{GHz}(802.11 \mathrm{a})$ input tones and an $\mathrm{LO}$ of $3.2 \mathrm{GHz}$. The intermodulation for tones that leak through the mixer was determined using a $5.7 \mathrm{GHz}$ and a $5.8 \mathrm{GHz}$ signal (two 802.11a interferers). The intermodulation product at 100 $\mathrm{MHz}$ showed an IIP2 $>+40 \mathrm{dBm}$, regardless of the LO frequency. The $\mathrm{LO}$ leakage to the RF input is below $-60 \mathrm{dBm}$ for $\mathrm{LO}$ frequencies up to $4 \mathrm{GHz}$ and below $-5 \mathrm{dBm}$ up to $7 \mathrm{GHz}$.

Fig. 15 shows a breakdown of the power consumption of the different parts of the implemented circuit. The I/Q-BLIXER core consumes $16 \mathrm{~mW}$ and the biasing and IF buffering combined consumes $13 \mathrm{~mW}$. Note that the IF buffers are added for measurements purposes and can often be omitted. At an LO frequency of $500 \mathrm{MHz}$, the $25 \%$ duty-cycle generation and $\mathrm{LO}$ buffering consume only $4 \mathrm{~mW}$. At an LO frequency of $7 \mathrm{GHz}$ this part consumes $28 \mathrm{~mW}$, which is almost half of the total power consumption. The LO buffering is based on inverters, which explains the increase in (dynamic) power consumption at higher $\mathrm{LO}$ frequencies.

In Table I the measured performance is summarized and compared to other state-of-the-art wideband down-converters. The I/Q-BLIXER achieves the widest signal bandwidth. Note that we

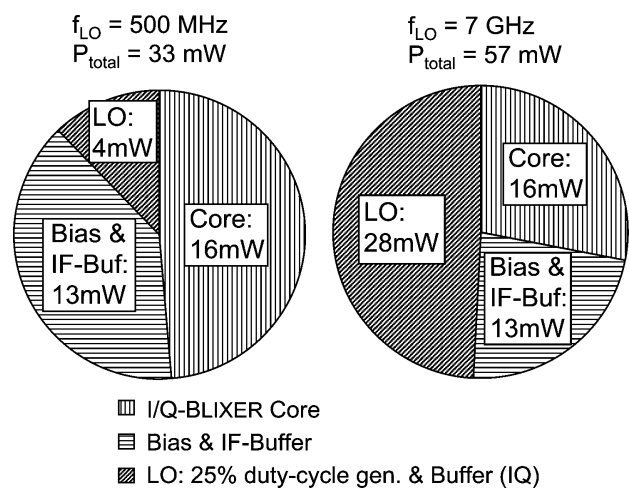

Fig. 15. I/Q-BLIXER power consumption breakdown for $500 \mathrm{MHz}$ and $7 \mathrm{GHz}$.

specify the $-1 \mathrm{~dB}$ bandwidth, where others often specify the $-3 \mathrm{~dB}$ bandwidth. The obtained linearity is the highest among the reference designs. The main reason for the high linearity is that the (RF) linearity is determined by only one transistor (combination), the CS inverter.

The noise figure is comparable to the other designs; furthermore, the NF is almost flat across the entire band. Note that this NF includes the PCB losses and that no external balun is needed.

Due to the absence of on-chip inductors, the area is very small. The combined area of the I/QBLIXER core and the I/Q-LO buffers is more than 4.5 times smaller than the smallest reference design.

For low LO frequencies, the power consumption of the LO buffering is much lower than the other designs, and for high LO frequencies, it is in the same order. The design of [20] has a lower core (LNA plus mixer) power consumption. However, in contrast to our design, this design has no quadrature outputs. Compared to the designs with quadrature outputs [1]-[3], the power consumption of our design is about 2 times lower.

\section{CONCLUSION}

This paper proposes the BLIXER topology, which stacks a current-commutating mixer on top of a noise-canceling balunLNA. The proposed topology has several attractive properties. It achieves a high and flat gain over a wide bandwidth without inductors for bandwidth extension. This is because the BLIXER directly converts the currents of the balun-LNA core to IF via current-commutating mixers, of which the (real part of) the input impedance is low. Creating voltage gain is shifted from RF to baseband where capacitive loading is not a problem. By using an I/Q mixer with $25 \%$ duty-cycle LO waveform, the output IF currents have also have a reduced duty-cycle, resulting in smaller DC-voltage drops after IF filtering. This allows for a 2 times increase of the impedance level of the IF load, rendering 2 times more voltage gain for the same supply headroom. The I/Q-BLIXER theoretically has only $1 \mathrm{~dB}$ less gain than a balun-LNA biased at the same current. Also, its double sided noise figure is only $1-2 \mathrm{~dB}$ higher than for the balun-LNA alone. The I/Q-BLIXER topology implements balun, LNA, and I/Q down-conversion functionality, all in one circuit core. A $65 \mathrm{~nm}$ implementation achieves $>18 \mathrm{~dB}$ conversion gain, a flat $\mathrm{NF}<5.5 \mathrm{~dB}$ from $500 \mathrm{MHz}$ to $7 \mathrm{GHz}, \mathrm{IIP} 2=+20 \mathrm{dBm}$ and IIP3 $=-3 \mathrm{dBm}$. The core circuit consumes $16 \mathrm{~mW}$ which 
TABLE I

COMPARISON OF STATE-OF-THE-ART WIDEBAND DOWN-CONVERTERS

\begin{tabular}{|c|c|c|c|c|c|c|}
\hline \multicolumn{2}{|c|}{ Parameter } & This Work & $\begin{array}{c}\text { Lee } \\
\text { ISSCC'07 [1] }\end{array}$ & $\begin{array}{c}\text { Craninckx } \\
\text { ISSCC'07 [2] }\end{array}$ & $\begin{array}{c}\text { Bagheri } \\
\text { JSSC'06 [3] }\end{array}$ & $\begin{array}{c}\text { Amer } \\
\text { JSSC'07 [20] }\end{array}$ \\
\hline \multicolumn{2}{|c|}{ RX Frequency [GHz] } & $0.5-7^{1)}$ & $2-8$ & $\begin{array}{l}1.8 \& \\
5-6\end{array}$ & $0.8-6$ & $0.1-3.85$ \\
\hline \multicolumn{2}{|c|}{ Gain [dB] } & $\begin{array}{c}18 \\
\text { excl. IF-Amp }\end{array}$ & $\begin{array}{c}23 \\
\text { incl. IF-Amp }\end{array}$ & $\begin{array}{c}10-90 \\
\text { incl. IF-Amp }\end{array}$ & $\begin{array}{c}3-36 \\
\text { incl. IF-Amp }\end{array}$ & $\begin{array}{c}20 \\
\text { incl. IF-Amp }\end{array}$ \\
\hline \multicolumn{2}{|c|}{ IIP3 [dB] } & -3 & -7 & -9 & -3.5 & $-3.2^{2)}$ \\
\hline \multicolumn{2}{|c|}{ IIP2 @ RF [dB] } & +20 & +18 & $?$ & $?$ & $?$ \\
\hline \multicolumn{2}{|c|}{$\mathrm{NF}[\mathrm{dB}]$} & $4.5-5.5$ & 4.5 & $4-8$ & $5-5.5$ & $\begin{array}{c}8.4-11.5 \\
\text { (SSB) }\end{array}$ \\
\hline \multicolumn{2}{|c|}{$\mathrm{S}_{11}[\mathrm{~dB}]$} & $<-10$ & $<-8$ & $<-9$ & $<-10$ & $<-10$ \\
\hline \multirow{2}{*}{ 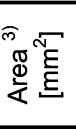 } & $\begin{array}{l}\text { LNA + } \\
\text { IQ-Mixers }\end{array}$ & $<0.01$ & \multirow{2}{*}{0.09} & 0.6 & 0.5 & $\begin{array}{c}0.88 \\
\text { (only I-path) }\end{array}$ \\
\hline & $\begin{array}{l}\text { LO Buffers } \\
(I \& Q)\end{array}$ & $<0.01$ & & 0.3 & $?$ & $?$ \\
\hline \multirow{2}{*}{ 市方 } & $\begin{array}{l}\text { LNA + } \\
\text { IQ-Mixers }\end{array}$ & 16 & 38 & $\begin{array}{c}34 \\
\text { WLAN setting }\end{array}$ & $\begin{array}{c}29 \\
(I \& Q ?)\end{array}$ & $\begin{array}{c}9.8 \\
\text { (only I-path) }\end{array}$ \\
\hline & $\begin{array}{l}\text { LO Buffers } \\
(I \& Q)\end{array}$ & $4-28$ & 24 & $\begin{array}{c}17 \\
\text { WLAN setting }\end{array}$ & $?$ & $?$ \\
\hline \multicolumn{2}{|c|}{ CMOS Technology } & $65 \mathrm{~nm}$ & $65 \mathrm{~nm}$ & $0.13 \mu \mathrm{m}$ & $90 \mathrm{~nm}$ & $90 \mathrm{~nm}$ \\
\hline \multicolumn{2}{|c|}{ VDD } & $1.2 \mathrm{~V}$ & $1.2 \mathrm{~V}$ & $1.2 \mathrm{~V}$ & $2.5 \mathrm{~V}$ & $1.2 \mathrm{~V}$ \\
\hline
\end{tabular}

1) This is $-1 \mathrm{~dB}$ bandwidth; others use $-3 \mathrm{~dB}$ bandwidth

2) Estimated using $P_{\| I P 3}=P_{1 d B C P}+9.6 d B$ [21]

3) Estimated from chip micrographs

is about 2 times lower than comparable wideband down-converters with I/Q-outputs. The area of the I/Q-BLIXER core occupies less than $0.01 \mathrm{~mm}^{2}$ in $65 \mathrm{~nm}$ CMOS, which makes it the smallest wideband down-converter design published.

\section{REFERENCES}

[1] S. Lee, J. Bergervoet, K. S. Harish, D. Leenaerts, R. Roovers, R. van de Beek, and G. van der Weide, "A broadband receive chain in $65 \mathrm{~nm}$ CMOS," in IEEE ISSCC Dig. Tech. Papers, Feb. 2007, pp. 418-612.

[2] J. Craninckx, M. Liu, D. Hauspie, V. Giannini, T. Kim, J. Lee, M. Libois, D. Debaillie, C. Soens, M. Ingels, A. Baschirotto, J. Van Driessche, L. P. Van der Perre, and P. Vanbekbergen, "A fully reconfigurable software-defined radio transceiver in $0.13 \mu \mathrm{m}$ CMOS," in IEEE ISSCC Dig. Tech. Papers, Feb. 2007, pp. 346-607.

[3] R. Bagheri, A. Mirzaei, S. Chehrazi, M. E. Heidari, M. Lee, M. Mikhemar, W. Tang, and A. A. Abidi, "An 800- MHz-6-GHz software-defined wireless receiver in 90-nm CMOS," IEEE J. Solid-State Circuits, vol. 41, pp. 2860-2876, Dec. 2006.

[4] S. C. Blaakmeer, E. A. M. Klumperink, B. Nauta, and D. M. W. Leenaerts, "An inductorless wideband balun-LNA in $65 \mathrm{~nm}$ CMOS with balanced output," in Proc. 33rd Eur. Solid-State Circuits Conf. (ESSCIRC), Sep. 2007, pp. 364-367.

[5] S. C. Blaakmeer, E. A. M. Klumperink, D. M. W. Leenaerts, and B. Nauta, "Wideband balun-LNA with simultaneous output balancing, noise-canceling and distortion-canceling," IEEE J. Solid-State Circuits, vol. 43, pp. 1341-1350, Jun. 2008.

[6] S. Chehrazi, A. Mirzaei, R. Bagheri, and A. A. Abidi, "A 6.5 GHz wideband CMOS low noise amplifier for multi-band use," in Proc. IEEE Custom Integrated Circuits Conf. (CICC'05), Sep. 2005, pp. 801-804.

[7] S. C. Blaakmeer, E. A. M. Klumperink, D. M. W. Leenaerts, and B. Nauta, "A wideband balun LNA I/Q-mixer combination in $65 \mathrm{~nm}$ CMOS," in IEEE ISSCC Dig. Tech. Papers, Feb. 2008, pp. 326-327.

[8] F. Bruccoleri, E. A. M. Klumperink, and B. Nauta, "Noise cancelling in wideband CMOS LNAs," in IEEE ISSCC Dig. Tech. Papers, Feb. 2002, pp. 406-407.
[9] F. Bruccoleri, E. A. M. Klumperink, and B. Nauta, "Wide-band CMOS low-noise amplifier exploiting thermal noise canceling," IEEE J. SolidState Circuits, vol. 39, pp. 275-282, Feb. 2004.

[10] F. Bruccoleri, E. A. M. Klumperink, and B. Nauta, Wideband Low Noise Amplifiers Exploiting Thermal Noise Cancellation. : Kluwer/ Springer, 2005

[11] J. Jussila and P. Sivonen, "A 1.2-V highly linear balanced noise-cancelling LNA in 0.13- $\mu \mathrm{m}$ CMOS," IEEE J. Solid-State Circuits, vol. 43, pp. 579-587, Mar. 2008.

[12] C. F. Liao and S. I. Liu, "A broadband noise-canceling CMOS LNA for 3.1-10.6-GHz UWB receivers," IEEE J. Solid-State Circuits, vol. 42, pp. 329-339, Feb. 2007.

[13] S. C. Blaakmeer, E. A. M. Klumperink, D. M. W. Leenaerts, and B. Nauta, "A wideband noise-canceling CMOS LNA exploiting a transformer," in Digest of Papers-IEEE Radio Frequency Integrated Circuits Symposium, 2006, pp. 137-140.

[14] V. J. Arkesteijn, "Analog Front-Ends for Software-Defined Radio Receivers," Ph.D., University of Twente, Enschede, 2007.

[15] V. J. Arkesteijn, E. A. M. Klumperink, and B. Nauta, "A wideband high-linearity RF receiver front-end in CMOS," in Proc. 30th Eur. Solid-State Circuits Conf. (ESSCIRC), Sep. 2004, pp. 71-74.

[16] H. Sjoland, A. Karimi-Sanjaani, and A. A. Abidi, "A merged CMOS LNA and mixer for a WCDMA receiver," IEEE J. Solid-State Circuits, vol. 38, pp. 1045-1050, Jun. 2003.

[17] J. Harvey and R. Harjani, "Analysis and design of an integrated quadrature mixer with improved noise, gain and image rejection," in IEEE International Symposium Circuits and Systems, May 2001, vol. 4, pp. 786-789.

[18] E. A. M. Klumperink, R. Shresta, E. Mensink, V. J. Arkesteijn, and B. Nauta, "Cognitive radios for dynamic spectrum access-polyphase multipath radio circuits for dynamic spectrum access," IEEE Cотmиnications Magazine, vol. 45, pp. 104-112, May 2007.

[19] B. Gilbert, "The MICROMIXER: A highly linear variant of the gilbert mixer using a bisymmetric class-AB input stage," IEEE J. Solid-State Circuits, vol. 32, pp. 1412-1423, Sep. 1997.

[20] A. Amer, E. Hegazi, and H. F. Ragaie, "A 90-nm wideband merged CMOS LNA and mixer exploiting noise cancellation," IEEE Journal of Solid-State Circuits, vol. 42, pp. 323-328, Feb. 2007.

[21] B. Razavi, RF Microelectronics. Upper Saddle River, NJ: Prentice Hall, 1988. 


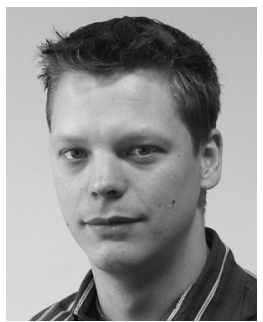

Stephan C. Blaakmeer (S'00-M'08) was born in Stiens, The Netherlands, in 1976. He received the M.Sc. degree in electrical engineering from the University of Twente, Enschede, The Netherlands, on the subject of RF CMOS ring oscillators, in 2001. He joined Ericsson Eurolab, Emmen, The Netherlands, in 2001, where he worked on CMOS radios for Bluetooth. In 2003, he returned to the IC Design group of the University of Twente to work toward the Ph.D. degree on the subject of wideband receiver techniques in CMOS

He recently joined Axiom IC, Enschede, The Netherlands, where he is working on RF transceivers. His interests are RF and analog circuits in general and more specific in circuits for wireless transceivers.

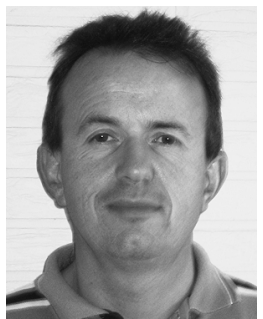

Eric A. M. Klumperink (M'98-SM'06) was born on April 4, 1960, in Lichtenvoorde, The Netherlands. He received the B.Sc. degree from HTS, Enschede, The Netherlands, in 1982. After a short period in industry, he joined the Faculty of Electrical Engineering of the University of Twente (UT) in Enschede in 1984, participating in analog CMOS circuit design and research. This resulted in several publications and a Ph.D. thesis, in 1997 ("Transconductance based CMOS circuits").

After receiveing his Ph.D., he started working on RF CMOS circuits. He is currently an Associate Professor at the IC-Design Laboratory which participates in the CTIT Research Institute (UT). He holds several patents and has authored or coauthored more than 80 journal and conference papers.

In 2006 and 2007, Dr. Klumperink served as an Associate Editor for IEEE TRANSACTIONS ON CIRCUITS AND SYSTEMS II, and since 2008 for IEEE TRANSACTIONS ON CIRCUITS AND SYSTEMS I. He was a corecipient of the ISSCC 2002 Van Vessem Outstanding Paper Award.

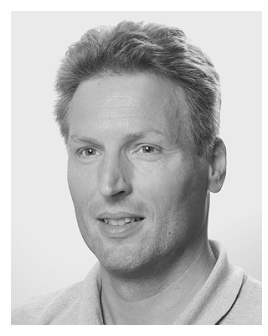

Domine M. W. Leenaerts (M'94-SM'96-F'2005) received the Ph.D. degree in electrical engineering from Eindhoven University of Technology, Eindhoven, The Netherlands, in 1992.

From 1992 to 1999, he was with Eindhoven University of Technology as an Associate Professor with the Micro-electronic Circuit Design group. In 1995, he was a Visiting Scholar with the Department of Electrical Engineering and Computer Science, University of California, Berkeley. In 1997, he was an Invited Professor with the Technical University of
Lausanne (EPFL), Lausanne, Switzerland. From 1999 until 2006, he was with Philips Research Laboratories. Since 2007, he has been with NXP Semiconductors, Research, Eindhoven, The Netherlands, as a Senior Principal Scientist, involved in (CMOS) RF integrated transceiver design. He has published over 150 papers in scientific and technical journals and conference proceedings and holds over 20 U.S. patents. He has coauthored several books, including Circuit Design for RF Transceivers (Kluwer, 2001).

Dr. Leenaerts served as IEEE Distinguished Lecturer in 2001-2003 and served as an associate editor of the IEEE TRANSACTIONS ON CIRCUITS AND SYSTEMS PART I (2002-2004). Since 2005, he has been the IEEE Circuits and Systems Society Member representative in the IEEE Solid-State Circuits Society Administrative Committee. Since 2007, he has served as an Associate Editor of the IEEE JOURNAL OF SOLID-STATE CIRCUITS. He is a member of the technical program committees of the ISSCC, ESSCIRC, and RFIC Conference.

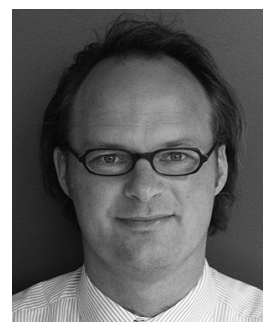

Bram Nauta (M'91-SM'03-F'08) was born in Hengelo, The Netherlands, in 1964. In 1987, he received the M.Sc. degree (cum laude) in electrical engineering from the University of Twente, Enschede, The Netherlands. In 1991, he received the $\mathrm{Ph} . \mathrm{D}$. degree from the same university on the subject of analog CMOS filters for very high frequencies.

In 1991, he joined the Mixed-Signal Circuits and Systems Department of Philips Research, Eindhoven, The Netherlands, where he worked on high-speed A/D converters and analog key modules. In 1998, he returned to the University of Twente as a full Professor heading the IC Design group, which is part of the CTIT Research Institute. His current research interest is high-speed analog CMOS circuits. He is also a part-time consultant in industry. In 2001, he cofounded Chip Design Works. His Ph.D. thesis was published as a book: Analog CMOS Filters for Very High Frequencies (Springer, 1993) and he received the Shell Study Tour Award for his Ph.D. work.

From 1997 until 1999, he served as an associate editor of IEEE TRANSACTIONS ON CIRCUITS AND SySTEMS PART II-ANALOG AND DigitaL SignAL PROCESSINg. After this, he served as a Guest Editor, an Associate Editor (2001-2006), and from 2007 as Editor-in-Chief for the IEEE JOURNAL OF SOLID-STATE CIRCUITS. He is also a member of the technical program committees of the IEEE International Solid State Circuits Conference (ISSCC), the European Solid State Circuit Conference (ESSCIRC), and the Symposium on VLSI Circuits. He was a corecipient of the ISSCC 2002 Van Vessem Outstanding Paper Award. He is a distinguished lecturer of the IEEE and elected member of IEEE SSCS AdCom. 